\title{
Análise das prescrições dispensadas em uma unidade básica de saúde do município de ljuí - RS
}

\author{
Vanessa Adelina Casali Bandeira1', Carina Talice Stube Hermann'1, \\ Carla Meggiolaro Siqueira ${ }^{1}$, Karla Renata de Oliveira ${ }^{2}$
}

\begin{abstract}
RESUMO
Avaliar as prescrições médicas, verificando Indicadores de Prescrição recomendados pela Organização Mundial da Saúde e aspectos legais vigentes no país. Trata-se de um estudo transversal, com análise das prescrições dispensadas em uma Unidade Básica de Saúde do município de ljuí-RS, através de um questionário como método indireto e quantitativo de coleta de dados. Foram avaliadas 4.108 prescrições, com média de 2,43 medicamentos/prescrição. 0 nome do paciente esteve presente em $94,9 \%$ das prescrições. Das informações referentes ao prescritor, a assinatura esteve em $99,3 \%$ e o carimbo e inscrição no Conselho Regional de Medicina em 88,9\%. Nas informações referentes ao medicamento as ausências foram: via de administração $(12,1 \%)$, forma farmacêutica $(10,2 \%)$, dose $(16,8 \%)$, posologia $(16,7 \%)$ e tempo de tratamento $(16,1 \%)$. Os resultados obtidos demonstram que os serviços de saúde no local do estudo encontram-se bem estruturados, apresentando-se de acordo com os critérios estabelecidos pela OMS e ao que preconiza a legislação.
\end{abstract}

Descritores: Prescrição de Medicamentos; Prescrições inadequadas; Erros de Medicação.

\section{Analysis of prescriptions dispensed in a basic health unit in the city of Ijuí/RS}

\begin{abstract}
The aim of this study was to evaluate medical prescriptions regarding the prescribing indicators recommended by the World Health Organization and the Brazilian current legislation. This cross sectional study analyzed the prescriptions dispensed in a Basic Health Unit in the city of ljuil RS through a questionnaire for data collection. It was evaluated 4.108 prescriptions, with a mean of 2.43 drugs by prescription. The patient's name was written in $94.9 \%$ of prescriptions. Regarding information related to the prescriber, the signature was present in $99.3 \%$ and the stamp and registration at the Regional Council of Medicine in $88.9 \%$. The information relating to drug showed the lack of: a) route of administration $(12.1 \%)$, b) pharmaceutical form $(10.2 \%)$, c) dose $(16.8 \%)$, d) dosage (16.7\%) and e) length of treatment (16,1\%). The results show that the health services are well organized and most prescriptions comply with the criteria established by WHO and Brazilian legislation.
\end{abstract}

Descriptors: Drugs Prescription; Prescribing Indicators; Medicine Errors; Dispensing.

${ }^{1}$ Farmacêutica pela Universidade Regional do Noroeste do Estado do Rio Grande do Sul (UNIJUÍ), ljuí, RS, Brasil.

${ }^{2}$ Mestre Farmacêutica, Docente do Departamento de Ciências da Vida da Universidade Regional do Noroeste do Estado do Rio Grande do Sul (UNIJUÍ), juí, RS, Brasil. 


\section{Introdução}

Asaúde é um direito fundamental do ser humano, sendo dever do Estado assegurar as condições de seu exercício, entre eles, o fornecimento de medicamentos ${ }^{1}$. Além disso, a Portaria $3.916^{2}$ e a Política Nacional de Medicamentos ${ }^{3}$ preconizam 0 acesso da população a medicamentos seguros, eficazes e de qualidade, através da prescrição medicamentosa e dispensação adequada dos medicamentos considerados essenciais ${ }^{1,2,3}$.

No Sistema Único de Saúde (SUS) o acesso da população aos medicamentos se dá através da prescrição emitida por profissional habilitado definida como uma ordem médica para fornecer um tratamento específico para determinado usuário do sistema ${ }^{4}$, constituindose em um documento legal, pelo qual são responsáveis os profissionais que prescrevem e dispensão a medicação 5 .

Diante disso, as prescrições devem seguir alguns critérios para garantir a adequada compreensão das informações que contêm, incluindo conforme a Lei $5.991^{6}$ legibilidade, identificação do usuário, nomenclatura oficial do fármaco prescrito por Denominação Comum Brasileira (DCB) e Denominação Comum Internacional (DCI), forma farmacêutica, posologia, via de administração, duração do tratamento e data de emissão da receita. Também deve apresentar os dados do prescritor como nome, assinatura, carimbo e número de inscrição no Conselho Regional de Medicina (CRM) .

De acordo com Ev, Guimarães \& Castro ${ }^{7}$ e Mastroianni ${ }^{8}$ através da análise das prescrições é possível caracterizar um serviço de saúde, pois reflete a atuação dos gestores, profissionais de saúde e usuários de medicamentos, demonstrando a qualidade dos serviços prestados e possibilitando que se construam alternativas que promovam o uso racional de medicamentos (URM) e consequentemente a organização dos serviços. Além disso, a Organização Mundial de Saúde (OMS) propõe indicadores capazes de avaliar o sistema de saúde através das prescrições, e verificar o uso racional de medicamentos ${ }^{9}$.

Alguns estudos demonstram que no Brasil as prescrições geralmente não se apresentam de acordo com os critérios estabelecidos, Guzatto \& Bueno ${ }^{10}$ ao analisarem as prescrições de uma Unidade Básica de Saúde (UBS) de Porto Alegre/RS encontraram que $18 \%$ das prescrições apresentavam pelo menos um tipo de erro. Valadão et al..$^{11}$ analisaram prescrições de uma UBS de Coronel Fabriciano/MG e observaram que 86,43\% continham pelo menos um erro.

Porém, essa situação não é exclusiva do Brasil, Khoja et al. ${ }^{12}$ verificaram que $18 \%$ das prescrições aviadas na atenção primária em Riyadh na Arábia Saudita continham erros. Zavaleta-Bustos et al. ${ }^{13}$ verificaram em um Centro de Saúde de uma Universidade no México a alta incidência de prescrições inapropriadas sendo que $58 \%$ apresentavam erros relacionados às doses recomendadas e à seleção do medicamento relacionado com a necessidade do paciente. Em Chicago nos Estados Unidos da América Bob et al. ${ }^{14}$ encontraram 64,2 erros a cada 1000 prescrições hospitalares analisadas.

Enfatiza-se que a prescrição é um instrumento fundamental na promoção do uso racional de medicamentos quando apresentada adequadamente, contribuindo para a dispensação adequada, a administração correta das doses, nos intervalos e tempo de tratamento adequados. Garantindo assim, que o consumo de medicamentos resulte na adesão adequada da terapêutica prescrita².

O objetivo deste estudo foi verificar em prescrições aviadas em uma UBS do município de ljuí/RS a presença das informações preconizadas pela legislação vigente no país e de alguns aspectos legais preconizados pela OMS.

\section{Metodologia}

Trata-se de um estudo transversal descritivo, com coleta de dados secundários em uma UBS do município de ljuí/RS. Foram incluídas no estudo as prescrições médicas, dispensadas no período de 2 de agosto de 2010 a 1 de setembro de 2010, que tiveram no mínimo um item dispensado.

Para a coleta de dados, utilizou-se um instrumento constituído de dezesseis questões a partir das quais foram obtidas informações referentes ao prescritor (data da prescrição, identificação do emitente e a sua especialidade), aos usuários (nome completo) e sobre os medicamentos (dosagem, forma farmacêutica, posologia, via de administração e duração do tratamento), assim como o nome do medicamento e o emprego da $\mathrm{DCB}^{6,2,15}$. Além disso, foram verificadas as informações referentes aos Indicadores de Prescrição da OMS no que se refere a média de medicamentos prescritos, porcentagem de medicamentos prescritos pelo nome genéricos, porcentagem de medicamentos prescritos pertencentes à Relação Municipal de Medicamentos (REMUME), porcentagem de antibióticos e porcentagem de injetáveis prescritos ${ }^{9}$.

Os medicamentos presentes nas prescrições foram classificados no primeiro nível do sistema de classificação Anatomical Therapeutic Chemical (ATC) do Nordic Council on Medicines, index $2010^{16}$. E a partir da REMUME, fornecida pela farmacêutica da Secretaria Municipal da Saúde de ljuí/RS, foi verificada a presença ou não de cada um na referida Relação. 
Quanto a legibilidade, foram consideras legíveis as prescrições cujas informações eram possíveis de serem compreendidas pelas pesquisadoras. Em relação a especialidade do prescritor, foi obtida a partir dos dados do carimbo e/ou na identificação do emitente e quando não informada, foi classificado como não especificada.

Foram seguidas as recomendações da Resolução n¹96/1996 do Conselho Nacional de Saúde, tendo sido obtida aprovação do Comitê de Ética em Pesquisa da Universidade Regional do Noroeste do Estado do Rio Grande do Sul (UNIJUÍ) sob o Parecer Consubstanciado n² 294/2010.

\section{Resultados}

Foram analisadas 4.108 prescrições médicas, entre as quais foram prescritos 10.101 medicamentos em média 2,43 medicamentos/prescrição ( $\pm 1,37$ medicamento), sendo que o número máximo de medicamentos prescrito por prescrição foi 11, verificado em duas prescrições.

Quanto aos aspectos legais e técnicos, verificou-se principalmente a ausência de carimbo e CRM do prescritor em $456(11,1 \%)$ das prescrições (Tabela 1).

Tabela 1 - Aspectos técnicos e legais relacionados ao paciente e ao médico prescritor presente nas prescrições analisadas em uma UBS de ljuí/RS.

\begin{tabular}{c|c|c}
\hline Aspectos técnicos e legais & $\mathbf{N}$ & $\%$ \\
\hline \multicolumn{3}{|c|}{ Nome do paciente } \\
\hline Sim & 3.899 & 94,9 \\
\hline Não & 9 & 0,2 \\
\hline Incompleto Carimbo e CRM do médico \\
\hline Sim & 3.652 & 11,1 \\
\hline Não & 456 & 88,9 \\
\hline Assinatura do médico & 200 & 11,1 \\
\hline Sim & 4.080 & 99,3 \\
\hline Não & 28 & 0,7 \\
\hline \multicolumn{3}{|c|}{ Legibilidade } \\
\hline Sim & 3.873 & 94,3 \\
\hline Não & 235 & 5,7 \\
\hline Sim & Data de emissão \\
\hline Não & 4.074 & 99,2 \\
\hline
\end{tabular}

Quanto as características relacionadas aos medicamentos, principalmente as referentes ao tratamento medicamentoso, apresentadas na Tabela 2, prevaleceu a ausência da dose do medicamento em 691 (16,8\%), seguido da posologia em $686(16,7 \%)$ das prescrições.

Entre as especialidades do médico prescritor a maioria não identificou sua especialidade, sendo observada essa situação em 2.278 (55,5\%) prescrições, seguido por clínico geral em 814 (19,8\%) prescrições (Figura 1).

Dos 10.101 medicamentos prescritos $9.572(94,8 \%)$ foram escritos de forma legível, dentre os quais $8.633(90,2 \%)$ foram prescritos pelo nome genérico, $8.995(94,0 \%)$ pertencem a REMUME, $233(2,4 \%)$ foram prescritos na forma injetável e $1.380(14,4 \%)$ eram antibióticos. 
Tabela 2 - Aspectos relacionados ao medicamento nas prescrições analisadas em uma UBS de ljuí/RS.

\begin{tabular}{c|c|c}
\hline $\begin{array}{c}\text { Características dos } \\
\text { medicamentos prescritos }\end{array}$ & $\mathbf{N}$ & $\%$ \\
\hline \multicolumn{3}{c}{ Via administração } \\
\hline Sim & 3.611 & 87,9 \\
\hline Não & 497 & 12,1 \\
\hline \multicolumn{3}{c}{ Forma farmacêutica } \\
\hline Sim & 3.691 & 89,8 \\
\hline Não Posologia & 10,2 \\
\hline Dose do medicamento \\
\hline Sim & 3.417 & 83,2 \\
\hline Não & 691 & 16,8 \\
\hline Sim & 3.422 & 83,3 \\
\hline Não & 686 & 16,7 \\
\hline Tempo de tratamento \\
\hline Sim & 3.446 & 83,9 \\
\hline Não & 662 & 16,1 \\
\hline
\end{tabular}

Figura 1 - Especialidade dos médicos prescritores.

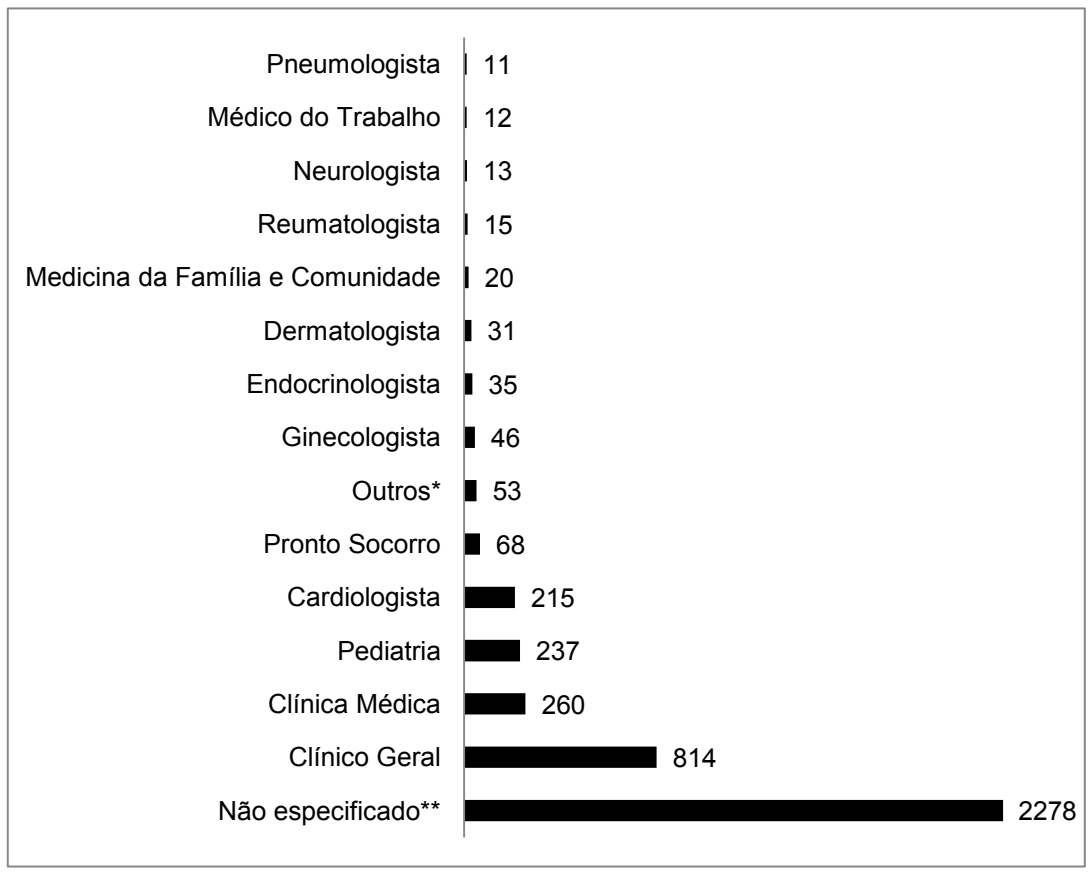

*Especialidades médicas presentes em menos de 10 prescrições

** Especialização não presente na prescrição.

Os medicamentos foram classificados no nível 1 do sistema ATC (Tabela 3). Além disso, os 15 medicamentos mais prescritos estão apresentados conforme denominação genérica na Figura 2, sendo produtos contendo ibuprofeno 0 medicamento mais prescrito, presente em 1.453 prescrições. 
Tabela 3: Classificação por grupo anatômico (ATC1) dos medicamentos prescritos durante o período do estudo.

\begin{tabular}{c|c|c}
\hline Medicamentos prescritos & $\mathbf{n}$ & $\%$ \\
\hline C - Sistema cardiovascular & 1.725 & 18,0 \\
\hline M - Sistema músculo esquelético & 1.553 & 16,3 \\
\hline N - Sistema nervoso & 1.550 & 16,3 \\
\hline J - Antiinfectantes gerais para uso sistêmico & 1.390 & 14,5 \\
\hline A - Aparelho digestivo e metabolismo & 1.316 & 13,8 \\
\hline R - Sistema respiratório & 776 & 8,1 \\
\hline H - Hormônios de uso sistêmico, excluindo & 602 & 6,3 \\
hormônios sexuais & & \\
\hline B - Sangue e órgãos hematopoiéticos & 347 & 3,6 \\
\hline D - Dermatológicos & 123 & 1,3 \\
\hline V - Vários & 85 & 0,9 \\
\hline S - Órgãos dos sentidos & 33 & 0,3 \\
\hline P - Produtos antiparasitários & 32 & 0,3 \\
\hline G - Sistema geniturinário e hormônios sexuais & 21 & 0,2 \\
\hline L - Antineoplásicos e agentes moduladores do & 7 & 0,1 \\
sistema imunológico & & \\
\hline Total & $9.560^{*}$ & 100,0 \\
\hline
\end{tabular}

* Excluídos os medicamentos que não foram encontrados na ATC e que não foram identificados pela falta de legibilidade.

Figura 2 - Os 15 medicamentos mais prescritos por denominação genérica.

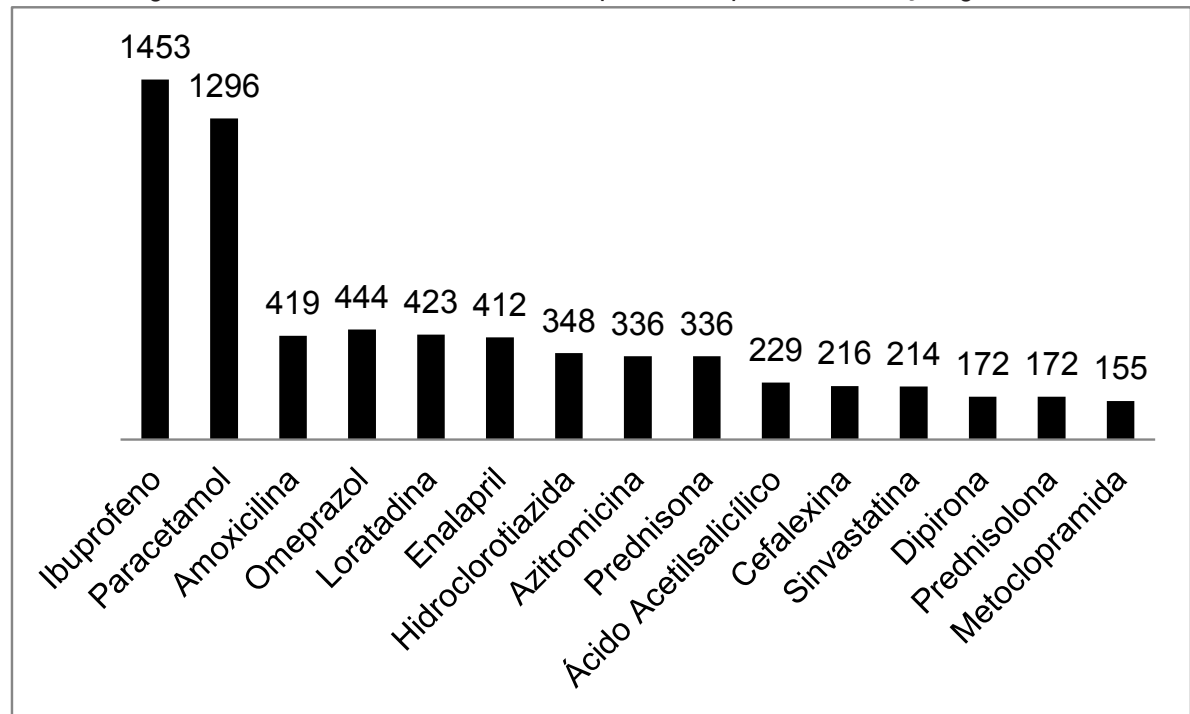

\section{Discussão}

A presença de erros em prescrições é frequente, indicando a necessidade de qualificação dos prescritores e à atuação direta do farmacêutico no suporte as decisões clínicas e na oferta de informações e orientações sobre os medicamentos, visando a redução de erros potencialmente prejudiciais aos usuários de medicamentos e a promoção de uma terapia medicamentosa segura e eficaz ${ }^{14}$.

Em um hospital da Inglaterra, farmacêuticos realizaram estudo prospectivo buscando as causas de erros nas prescrições, entre as principais identificaram a falta de atenção dos médicos aos prescrever, a sobrecarga do ambiente de trabalho, falta de comunicação na equipe, falta de conhecimento, hierarquia da equipe médica e ausência de autoconsciência de erros, reforçando a necessidade de treinamento dos prescritores ${ }^{17}$.

No presente estudo, verificou-se a ausência de informações referentes ao prescritor, ao usuário e ao medicamento, o que pode resultar em erros de medicação ou dificultar a comunicação entre os responsáveis pelo tratamento medicamentoso, e contribuir para a falta de eficácia ou segurança na terapia medicamentosa adotada. 
Ao verificar os aspectos legais da prescrição, o nome do usuário foi a variável mais presente, semelhante ao evidenciado por Bontempo et al. ${ }^{18} \mathrm{em}$ Contagem/MG de 99,6\%, Valadão et al. ${ }^{11}$ encontraram o nome do usuário em todas as prescrições de uma UBS de Coronel Fabriciano/MG, demonstrando que esse item geralmente encontra-se presente, o que facilita a oferta de informações ao usuário.

No que se refere às informações relacionadas ao médico prescritor Bontempo et al. ${ }^{18}$ verificaram que a assinatura estava presente em $99,6 \%$ das prescrições, estando de acordo com o presente estudo (99,3\%). No entanto, a presença do carimbo e CRM foi de $88,90 \%$ no presente estudo, diferente do estudo de Mastroianni8, realizado em Araraquara/ SP que verificou a presença do carimbo em $84,1 \%$ e o CRM 97\% e também de Valadão et al. ${ }^{19}$ no qual esses aspectos estiveram ausentes em $22 \%$ das prescrições.

A presença dos aspectos relacionados ao médico é fundamental, conforme a RDC $357^{15}$ a prescrição somente pode ser aviada pelo farmacêutico se nela constarem informações que possibilitem identificar o médico prescritor. Além disso, auxiliam na detecção de fraudes e irregularidades cometidas por médicos. A mesma Resolução apresenta a data como um item indispensável, pois ela indica o tempo decorrente entre a consulta e o acesso ao medicamento, sendo também determinante para a dispensação de medicamentos presentes na Portaria $344^{20}$ e RDC $22^{21}$, que vigem respectivamente sobre os medicamentos sujeitos a controle especial e os antimicrobianos. No presente estudo, salienta-se que a presença da data em $99,17 \%$ das prescrições é um aspecto positivo, diferente do estudo de Mastroianni ${ }^{8}$ presente em $87,4 \%{ }^{15,20,21}$.

Em relação a legibilidade $94,28 \%$ das prescrições analisadas foram consideradas legíveis, diferindo do estudo de Mastroianni $^{8}$ no qual apenas $58,8 \%$ puderam ser classificadas desta forma e Silvério \& Leite ${ }^{22}$ na cidade de Muiaé/MG que encontraram $36 \%$ das prescrições analisadas ilegíveis. A falta de legibilidade compromete o resultado da terapia medicamentosa, sendo que a legislação vigente estabelece que a legibilidade das prescrições é indispensável para a adequada compreensão e para a realização da dispensação do medicamento ${ }^{2,15}$.

A amostra em estudo apresentou uma média de 2,43 medicamentos/prescrição assemelhando-se a outros estudos, como 0 de Ev, Guimarães \& Castro ${ }^{7}$ realizado em Ouro Preto/MG e Silvério \& Leite ${ }^{22}$ em Muiaé/MG, ambos com média de 2,2 medicamentos. Na cidade de Porto Alegre/RS Guzatto \& Bueno $0^{10}$ encontraram em média 2,75 medicamentos/prescrição. Destaca-se ainda que o presente estudo evidenciou uma média um pouco superior ao que estabelece a OMS para a atenção básica ${ }^{23}$. Neste contexto, salienta-se que a média de medicamentos pode indicar o uso excessivo dos mesmos, resultando em polifarmácia e maior suscetibilidade a reações adversas e problemas relacionados a medicamentos.

Em relação aos itens que influenciam no tratamento observou-se resultados melhores do que os encontrados por outros autores, Bontempo et al. ${ }^{18}$ verificaram a presença da via de administração em $78,1 \%$ das prescrições, a forma farmacêutica em $58,1 \%$, a posologia em $79 \%$, o tempo de tratamento em $72,4 \%$ e a dose em $69,3 \%$. Para Valadão et. al. ${ }^{19}$ a via de administração esteve ausente em $27 \%$ das prescrições, a forma farmacêutica em $12 \%$, a dose em $67 \%, 0$ tempo de tratamento em $79 \%$ e a posologia em $1 \%$.

A ausência destes aspectos pode incorrer em erros que podem comprometer a interpretação da prescrição por parte dos dispensadores e a dispensação dos medicamentos ${ }^{22}$. Os autores sugerem que o farmacêutico como profissional do medicamento, avalie a prescrição médica e dispense quando as informações estiverem claras o suficiente para assegurar o correto tratamento, e quando não for possível a compreensão da prescrição, que este se comunique com o profissional prescritor para o esclarecimento das dúvidas. Ainda segundo estes autores, os erros de prescrições e as prescrições incompletas impedem a eficácia no trabalho de dispensação dos medicamentos, colocando em risco a qualidade da assistência farmacêutica e consequentemente no sucesso da terapia medicamentosa.

Em relação aos Indicadores de Prescrição da OMS, no estudo de Farias et al. ${ }^{24}$ realizado nas UBSs de Campina Grande/PB $84,2 \%$ dos medicamentos foram prescritos pelo nome genérico, $21,1 \%$ eram antibióticos e 1,1\% injetáveis e ainda, $91,9 \%$ constam na lista de medicamentos padronizados. Já no estudo Girotto \& Silva $(2006)^{25}$ realizado no município de Ibiporã/PR 70,2\% dos medicamentos foram prescritos pelo nome genérico, 22\% eram antibióticos, $7 \%$ injetáveis e 58,4\% encontravam-se na REMUME. No presente estudo o nome genérico esteve presente em 94,76\%, encontrando-se próximo ao recomendado pela OMS que é de $100 \%{ }^{23}$, o que facilita 0 acesso, pois permite a aquisição de medicamento de referência, genéricos ou similares.

Além disso, quanto a presença na REMUME, 93,97\% dos medicamentos prescritos pertencem a referida relação, sendo quea OMS recomenda que pelo menos $70 \%$ dos medicamentos prescritos estejam incluídos ${ }^{23}$, indicando que o sistema de saúde do município está organizado buscando atender a demanda de medicamentos de seus usuários, tendo em vista que muitos medicamentos presentes na REMUME não constam na Relação Nacional de Medicamentos (RENAME), sendo financiado pelo município. 
A porcentagem de antibiótico foi de $14,42 \%$, diferindo de Farias et al. ${ }^{24}$ e Girotto \& Silva ${ }^{25}$ que encontraram $21,1 \%$ e $22 \%$ respectivamente. No entanto, o percentual encontrado no presente estudo está de acordo com o que recomenda a OMS, no máximo $20 \%{ }^{23}$, embora a coleta de dados tenha sido realizada no inverno, período em que se observa um aumento no consumo destes medicamentos. Nesse contexto, observa-se que a baixa porcentagem de uso de antimicrobianos é importante, pois o uso irracional destes medicamentos tem consequências médicas e ecológicas, resultando em custos sociais e pessoais quando o uso é inadequado, que envolve custos diretos no tratamento, interações medicamentosas, podendo ocasionar em complicações na saúde do usuário ${ }^{26}$, e facilita a ocorrência de resistência bacteriana.

A forma farmacêutica injetável esteve presente em $2,43 \%$ das prescrições analisadas, estando de acordo com os $10 \%$ recomendado pela $\mathrm{OMS}^{23}$, sendo importante salientar que este resultado está relacionado principalmente a prescrição de insulinas NPH e Regular disponibilizadas pelo município.

Verificou-se prevalência dos medicamentos que atuam no sistema cardiovascular, indicando que a população que utiliza esse serviço de saúde é portadora de doenças crônicas do aparelho cardiovascular como hipertensão arterial sistêmica. Diferenciando-se de Colombo et al. ${ }^{27}$ que encontrou os medicamentos que atuam no sistema nervoso com maior frequência entre as prescrições e de Farias et al. ${ }^{24}$ que encontrou prevalência de antimicrobianos.

Considerando os medicamentos dispensados individualmente verificou-se que ibuprofeno foi o mais prescrito, seguido pelo paracetamol sendo que ambos pertencem a classe dos antiinflamatórios não-esteróides (AINEs), que representam um dos grupos de fármacos mais utilizados no mundo ${ }^{28}$, representando $27,21 \%$ dos medicamentos prescritos, semelhante aos resultados obtidos por Colombo et. al.27 que identificaram diclofenaco e paracetamol, ambos AINEs como os mais prescritos, e do estudo de Ev, Guimarães \& Castro $^{7}$ onde analgésicos antiespasmódicos e antiinflamatórios foram encontrados com maior frequência nas prescrições com $20,92 \%$.

Os AINEs apresentam atividades analgésica, antipirética, anti-inflamatória e antitrombótica, atuando através da inibição da síntese de prostaglandinas no organismo, sendo que são utilizados como medicamento para o tratamento assintomático inespecífico, constituindo-se em medicamentos com uso muito difundido no mundo ${ }^{28}$. No entanto, segundo Mota et al..$^{29} \mathrm{O}$ uso indiscriminado desses medicamentos pode representar riscos a saúde dos usuários, pois os mesmos podem resultar em interações medicamentosas e causar efeitos adversos como irritação na mucosa gastrointestinal inferior, lesão hepática, indução de neutropenia, efeitos na pressão arterial, efeitos pulmonares, inibição plaquetária, reações de hipersensibilidade, efeitos no sistema nervoso central e efeitos em cartilagens ${ }^{30}$, quando utilizados de forma incorreta com superdose ou por tempo indevidamente prolongado. Além disso, segundo Alonzo \& Corrêa ${ }^{31}$ os medicamentos correspondem a segunda causa de maior frequência de intoxicação no Brasil, e os analgésicos são responsáveis por cerca de $10 \%$ dessas intoxicações, bem como os principais medicamentos responsáveis por intoxicações acidentais por crianças e na tentativa de suicídio de adultos. Além disso, de acordo com Centro de Informação Tecnológica do Rio Grande do Sul², os AINEs foram responsáveis por 440 intoxicações no Estado, destas 220 foram causadas pelo uso de ibuprofeno.

Entre as limitações do presente estudo estão o local do estudo, tendo em vista que a pesquisa foi realizada concentrada em apenas uma UBS do município, sendo a mesma a UBS central e com grande fluxo de usuários, e o pequeno período pesquisado, no entanto, permite observar um panorama da situação do local analisado. Além disso, a falta de informações sobre os usuários de medicamentos e a compreensão dos mesmos sobre a prescrição impossibilitam mensurar detalhadamente 0 que os erros e omissões das prescrições influenciam no resultado da terapia medicamentosa destes sujeitos.

Os resultados obtidos reforçam a necessidade de se tratar a prescrição como um documento terapêutico, sendo dessa forma um instrumento efetivo para assegurar o URM prevenindo erros e abuso do uso dos medicamentos. Para isso, profissionais prescritores e dispensadores devem estar cientes de suas responsabilidades diante da legislação sanitária e profissional ${ }^{8}$, sendo crucial a atuação preventiva de erros ${ }^{12}$. Para isso, se faz necessária a qualificação e capacitação dos prescritores para a elaboração de prescrições adequadas, baseadas na identificação do problema do usuário, no objetivo terapêutico, na seleção do medicamento por eficácia, segurança e custo, escrita de forma correta, assegurando o uso apropriado dos medicamentos ${ }^{33}$.

Além disso, a atuação dos profissionais farmacêuticos é fundamental na identificação dos erros das prescrições no ato da dispensação, auxiliando na promoção do URM. Oenning, Oliveira \& Blatt ${ }^{34}$ ao verificar o conhecimento dos usuários de medicamentos após consulta médica e dispensação no município de Grão Pará/SC verificaram que os resultados foram melhores após a dispensação do que após a consulta médica, destacando a importância do farmacêutico na orientação para a correta utilização de medicamentos. 
A prescrição é um instrumento norteador do URM, a qual deve promover a interação entre paciente, médico e farmacêutico, sendo que a qualidade dessa interação está diretamente relacionada ao resultado terapêutico ${ }^{5}$. Neste contexto o farmacêutico representa o último elo entre o serviço de saúde e o usuário de medicamento, sendo o profissional capacitado para identificar erros e tentar solucioná-los para assim fornecer o tratamento medicamentoso correto.

Além disso, uma contribuição mais efetiva do farmacêutico pode ocorrer através da oferta do serviço de Atenção Farmacêutica (AF), prática que promove o contato direto do farmacêutico com o usuário, visando melhorar os resultados da terapia medicamentosa e consequentemente a qualidade de vida do mesmo. No entanto, Fegadolli et al..$^{35}$, avaliaram a implementação da AF e concluíram que para uma efetiva implementação em diversos ambientes de trabalho é necessário investimento em formação e capacitação dos profissionais, bem como aceitação do público e interação com a equipe de saúde envolvida.

Com isso, a atuação do profissional farmacêutico em uma equipe na atenção básica é de extrema importância, pois além de ser o profissional do medicamento quando inserido na gestão e organização do sistema de saúde é capaz de contribuir para que através de uma equipe multiprofissional seja possível se estabelecer uma adequada comunicação entre os profissionais, podendo ser a prescrição médica um instrumento que possibilite a adequada comunicação, promovendo a melhora no funcionamento do sistema e na qualidade de vida dos seus usuários.

\section{Considerações Finais}

Com os resultados obtidos através da análise das prescrições, podemos observar que os serviços de saúde no local do estudo encontram-se bem estruturados, apresentando-se de acordo com os critérios estabelecidos pela OMS e a maioria das prescrições atende ao que preconiza a legislação. Com isso, verifica-se um acesso mais seguro e de qualidade dos usuários do sistema ao tratamento medicamentoso.

Embora os resultados obtidos indiquem que o local do estudo cumpre de forma satisfatória com o que preconizam a legislação vigente e a OMS, sugere-se a qualificação constante dos profissionais de saúde para aperfeiçoar e sanar os erros ainda presentes e garantir um tratamento medicamentoso adequado aos usuários desse serviço de saúde.

Nesse contexto, o farmacêutico contribui em todas as etapas do processo para a promoção de uma terapia medicamentosa adequada, desde a seleção dos medicamentos mais utilizados para a inclusão nas listas padronizadas, no armazenamento e distribuição dos medicamentos até a oferta de orientações sobre os medicamentos aos usuários, principalmente quando inserido em uma equipe interdisciplinar que busca desenvolver suas atividades de forma adequada para obter os resultados desejados.

\section{Referências Bibliográficas}

1. Brasil. Lei n. 8.080, de 19 de Setembro de 1990. Dispõe sobre as condições para a promoção, proteção e recuperação da saúde, a organização e o funcionamento dos serviços correspondentes e dá outras providências. Presidente da República. Brasília, 19 set 1990. Disponível em: URL http://portal.saude.gov.br/portal/arquivos/pdf/LEl8080.pdf.

2. Brasil. Ministério da Saúde. Portaria n. 3.916/GM, de 30 de outubro de 1998. Aprova a política nacional de medicamentos, cuja íntegra consta no anexo desta portaria. (ementa elaborada pela CDI/MS). Diário Oficial da União, Brasília, 30 out 1998. Disponível em: URL http://portal.saude.gov.br/portal/arquivos/pdf/portaria3916_30out98.pdf.

3. Brasil. Ministério da Saúde, Secretaria de Políticas de Saúde, Departamento de Atenção Básica. Política Nacional de Medicamentos; 2001. Disponível em: URL http://bvsms.saude.gov.br/bvs/publicacoes/politica_medicamentos.pdf.

4. Lofholm PW, Katzung BG. Prescrição Racional \& Elaboração da Prescrição. In: Katzung BG. Farmacologia Básica e Aplicada. $8^{a}$ ed. Rio de Janeiro: Editora Guanabara Koogan S. A; 2003. p. 957-964.

5. Pepe VLE, Castro CGSO. A interação entre prescritores, dispensadores e pacientes: informação compartilhada como possível benefício terapêutico. Cad Saúde Pública 2000; 16(3):815-822.

6. Brasil. Presidente da República. Lei n. 5.991, de 17 de dezembro de 1973. Dispõe sobre o controle sanitário do comércio de drogas, medicamentos, insumos farmacêuticos e correlatos, e dá outras providências. Diário Oficial da União, Brasília, 19 dez 1973. Disponível em: URL http://www.cff.org.br/userfiles/file/leis/5991.pdf.

7. Ev LS, Guimarães AG, Castro VS. Avaliação das Prescrições Dispensadas em uma Unidade Básica de Saúde do Município de Ouro Preto, Minas Gerais, Brasil. Latin American Journal of Pharmacy 2008; 27(4):543-547. 
8. Mastroianni PC. Análise dos aspectos legais das prescrições de medicamentos. Rev Ciênc Farm BásicaApl 2009; 30(2):173-176. 9. World Health Organization. How to investigate drug use in health facilities - selected drug use indicators. Geneva, WHO; 1993. Disponível em: URL http://apps.who.int/medicinedocs/pdf/s2289e/s2289e.pdf.

10. Guzatto P, Bueno D. Análise de prescrição medicamentosas dispensadas na Farmácia de uma Unidade Básica de Saúde de Porto Alegre - RS. Rev HCPA 2007; 27(3):20-26.

11. Valadão AF, Moreira ALP, Andrade LC, Pires CA, Firmino KF, Brum CA. Prescrição médica: um foco nos erros de prescrição. Rev Bras Farm 2009; 90(4):340-343.

12. Khoja T,Neyaz Y,Qureshi NA,Magzoub MA,Haycox A,Walley T. Medication errors in primary care in Riyadh City, Saudi Arabia. East Mediterr Health J 2011; 17(2):156-159.

13. Zavaleta-Bustos M, Castro-Pastrana, Reyes-Hernández I, Lópes-Luna MA, Bermúdez-Camps IB. Prescrtiption errors in a primary care university unit: urgency of pharmaceutical care in Mexico. Rev Bras Cienc Farm 2008; 44(1):115-125.

14. Bob A, Gleason K, Husch M, Feinglass J, Yarnold PR, Noskin GA. The Epidemiology of Prescribing Errors. Arch Intern Med 2004;164:785-792.

15. Brasil. Conselho Federal de Farmácia. Resolução n. 357. de 20 de abril de 2001a. Aprova o regulamento técnico das Boas Práticas de Farmácia. Diário Oficial da União. Disponível em: URL http://www.prosaude.org.br/legislacao/119Resolu\%C3\%A7\%C3\%A30\%20CFF\%20n\%C2\%BA\%20357\%20de\%2020-04-1.doc.

16. World Health Organization. Collaborating Centre for Drug Statistics Methodology. Anatomical Therapeutic Chemical ATC/DDD Index, 2010. Disponivel em: URL/http://www.whocc.no/atc_ddd_index/.

17. Dean B, Schachter M, Vicent C, Baber N. Causes of prescribing errors in hospital inpatients: a prospective study. Lancet 2002; 359:1373-1378.

18. Bontempo VL, Coelho MC, Chaver JG, Pereira LAM, Braccini CM. Análise das prescrições geradas pelas equipes do PSF e das Unidades de Atendimento Imediato, em dois distritos sanitários do município de Contagem/MG. Bol Pneumol Sanit 2003; 11(2):17-24.

19. Valadão AF, Firmina KF, Moreira ALP, Castro GD, Loures GF, Públio RN, Sena MPT, Brum CA. Estudo das prescrições de medicamentos nas Unidades Básicas de Saúde de Coronel Fabriciano - MG, quanto aos preceitos legais. Rev Bras Farm 2008; 89(4):298-301.

20. Brasil. ANVISA - Agência Nacional de Vigilância Sanitária. Portaria n. 344, de 12 de maio de 1998. Aprova 0 Regulamento Técnico sobre substâncias e medicamentos sujeitos a controle especial. Diário Oficial da União. Brasília, 15 mai 1998. Disponível em: URL <http://elegis.anvisa.gov.br/leisref/public/showAct.php?id=20054\&word=\#'>.

21. Brasil. ANVISA-Agência Nacional de Vigilância Sanitária. RDC n. 22, de 26 de outubro de 2010. Dispõe sobre o controle de medicamentos à base de substâncias classificadas como antimicrobianos, de uso sob prescrição médica, isoladas ou em associação e dá outras providências. Diário Oficial da União. Brasília, 26 out 2010. Disponível em: URL http://portal. anvisa.gov.br/wps/wcm/connect/c13443804478bef68eeff7d15359461/resolucao+antibioticos.pdf?MOD=AJPERES.

22. Silvério MS, Leite ICG. Qualidade das prescrições em município de Minas Gerais: uma abordagem farmacoepidemiológica. Rev Assoc Med Bras 2010; 56(6):675-80.

23. Organização Pan-Americana de Saúde. Ministérioo da Saúde. Avaliação da Assistência Farmacêutica no Brasil. Brasília, 2005. 24. Farias AD, Cardoso MAA, Medeiros ACD, Belém LF, Simões MO. Indicadores de prescrição médica nas unidades básicas de Saúde da Família do município de Campina Grande, PB. Rev Bras Epidemiol 2007; 10(2):149-156.

25. Girotto E, Silva, PV. A prescrição de medicamentos em um município do Norte do Paraná. Rev Bras Epidemiol 2006; 9(2):226-234. 26. Abrantes PM, Magalhães SMS, Acúrcio FA, Sakurai E. Avaliação da qualidade das prescrições de antimicrobianos dispensadas em unidades públicas de saúde de Belo Horizonte, Minas Gerais, Brasil, 2002. Cad Saúde Pública 2007; 23(1):95-104.

27. Colombo D, Helena ETS, Agostinho ACMG, Didjurgeit JSMA. Padrão de Prescrição de Medicamentos nas Unidades de Programa de Saúde da Família de Blumenau. Rev Bras Cienc Farm 2004; 40(4):549-558.

28. Wannmacher L, Bredemeier, M. Antiinflamatórios não-esteróides: Uso indiscriminado de inibidores seletivos de cicloxigenase-2. Ministério da Saúde 2004; 1(2):1-6.

29. Mota PM, Lima ALZ, Coelho E, Paula EMX, Furini AAC. Estudo sobre a utilização de antiinflamatórios não esteroidais prescritos em receitas para idosos da região Noroeste Paulista. Rev Ciênc Farm Básica Apl 2010; 31(2):157-163.

30. Kraychete D. Antiinflamatórios não-homonais. In: Silva P. Farmacologia. $6^{2}$ ed. Rio de Janeiro: Editora Guanabara Koogan S. A; 2002. p.556-565. 
31. Alonzo HGA, Corrêa CL. Analgésicos, antipiréticos e antiinflamatórios não-esteroidais dados epidemiológicos em seis centros de controle de intoxicação do Brasil. Rev bras toxicol 2001; 14(2):49-54.

32. Centro de Informações Toxicológica do Rio Grande do Sul. Relatório Anual de Atendimento - 2010. Disponível em: URL http://www.cit.rs.gov.br/images/stories/rel_2010.pdf.

33. Hogerzeil HV, Barnes KI, Henning RH, Kocabasoglu YE, Moller H, Smith AJ et al. Guia do Instrutor em Práticas da Boa Prescrição Médica. Organização Mundial da Saúde. Departamento de Medicamentos Essenciais e Políticas de Medicamentos. Genebra, Suíça: 2001.

34. Oenning D, Oliveira BV, Blatt CR. Conhecimento dos pacientes sobre os medicamentos prescritos após consulta médica e dispensação. Ciência \& Saúde Coletiva 2011; 16(7):3277-3283.

35. Fegadolli C, Santos DR, Fonseca DC, Marques TC. A percepção de farmacêuticos acerca da possibilidade de implantação da atenção farmacêutica na prática profissional. Revista Espaço para a Saúde 2010; 12(1):48-57.

\section{Vanessa Adelina Casali Bandeira}

Endereço para correspondência - Rua do Comércio, $n^{\circ}$ 3000, Bairro : Universitário, CEP: 98700-000, Cidade: ljuí, RS, Brasil.

E-mail: vanessa.acbandeira@yahoo.com.br

Lattes: http://lattes.cnpq.br/4383767989785264

Carina Talice Stube Hermann - carina.herman@unijui.edu.br

Carla Meggiolaro Siqueira - carlameggiolarosiqueira@yahoo.com.br

Karla Renata de Oliveira - karla@unijui.edu.br

\section{Enviado em 15 de setembro de 2014. Aceito em 30 de maio de 2015.}

